\title{
Effect of quercetin glucosides from Allium extracts on HepG2, PC-3 and HT-29 cancer cell lines
}

\author{
YINGKUN PAN $^{1}$, YI MEI ZHENG ${ }^{2}$ and WING SHING $\mathrm{HO}^{1}$ \\ ${ }^{1}$ School of Life Sciences, The Chinese University of Hong Kong, Shatin, 011-852 Hong Kong, SAR; \\ ${ }^{2}$ Minnan Normal University, College of Biological Science and Technology, Zhangzhou, Fujian 363000, P.R. China
}

Received July 3, 2015; Accepted October 13, 2016

DOI: $10.3892 / \mathrm{ol} .2018 .7893$

\begin{abstract}
The present study investigated the effects of quercetin glucosides, which were isolated from the Chinese onion (Allium chinense), garlic (Allium sativum), onion (Allium cepa $\mathrm{L}$.) and the Welsh onion (Allium fistulosum L.) in HepG2, HT-29 and PC-3 cancer cell lines. Quercetin-3,4'-di-O-glucoside $\left(3,4^{\prime}-\mathrm{Qdg}\right)$ and quercetin-4'-O-glucoside (4'-Qmg) comprise $\sim 98 \%$ of the flavonoids in the methanol extract of onion. A small amount of 3,4'-Qdg is present in the Welsh onion and Chinese onion, whereas 4'-Qmg is also present in the Welsh onion. In HepG2 cells, 4'-Qmg was demonstrated to exhibit more significant growth inhibition compared with 3,4'-Qdg and quercetin 3- $\beta$-D-glucoside, but exhibited less inhibitory effects in PC-3 and HT-29 cells. These results suggest the anti-proliferative potential of 4'-Qmg in various cancer cell lines and the health benefits of the genus Allium. The findings indicate the potential of 4'-Qmg as an anticancer agent for further development.
\end{abstract}

\section{Introduction}

The onion is the most widely cultivated species of the genus Allium. Flavonoids are found in onions, with notable bioactivity $(1,2)$. These plant-derived polyphenols serve beneficial roles in humans $(3,4)$ and contain chemical substances that irritate the eyes (1). Certain phenolic compounds and flavonoids exhibit anti-inflammatory, anti-cholesterol, anticancer and antioxidant properties (1-4). These plant-derived phytochemical flavonols include quercetins $(3,5)$ and quercetin glycosides (6,7). Quercetins have been reported to exhibit anticancer and anti-inflammatory activities $(8,9)$. The composition of flavonols in genus Allium varies widely depending on the growing conditions and storage (10). Previous animal studies have revealed that quercetin enhanced the antioxidant levels in the plasma (11-14). Quercetin derivatives have been used in the

Correspondence to: Professor Wing Shing Ho, School of Life Sciences, The Chinese University of Hong Kong, Mong Man Wai 604, Shatin, 011-852 Hong Kong, SAR, P.R. China

E-mail:ws203ho@cuhk.edu.hk

Key words: quercetin glucoside, Allium, apoptosis, cancer cells treatment of peroxynitrite-oxidized sarcoplasmic/endoplasmic reticulum calcium ATPase 1 (SERCA1) (15). In addition, the quercetin glucosides are more effectively absorbed than quercetin aglycone in humans $(11,16)$. The difference in absorption rate was attributed to glycosylation in quercetin metabolism. Previous studies have revealed that onions contain an abundant amount of flavonol glycosides as quercetin derivatives $(9,17)$. Flavonoids have been used as a diuretic agent and anti-inflammatory in folk medicine and certain flavonoid-rich types of onion exhibit anticancer properties (18-20). However, detailed research into the anticancer properties of quercetin has not yet been performed. The aim of the present study was to examine the composition of quercetins in onions and evaluate their effects in cancer cell lines.

\section{Materials and methods}

Chemicals and reagents. Quercetin-3,4'-di-O-glucoside (3,4'-Qdg; $\geq 98.5 \%$; Fig. 1A) and quercetin-4'-O-glucoside (4'-Qmg; $\geq 95 \%$; Fig. 1B) were purchased from Extrasynthese (Genay, France). Quercetin 3- $\beta$-D-glucoside (3-Qmg; $\geq 98 \%$; Fig. 1C) and rutin ( $\geq 95 \%$; Fig. 1D) were purchased from Tauto Biotech Co., Ltd. (Shanghai, China). All the solvents and chemicals are of analytical grade and were purchased from Thermo Fisher Scientific, Inc. (Waltham, MA, USA).

High performance liquid chromatography (HPLC) analysis. HPLC analysis was conducted using an Agilent 1100 HPLC system with the G1315A module Diode Array Detector (Agilent Technologies, Inc., Santa Clara, CA, USA) and with Alltech ${ }^{\circledR}$ Alltima $^{\mathrm{TM}}$ reversed-phase C18 HPLC columns $(4.6 \times 250 \mathrm{~nm}$, $5 \mu \mathrm{m}$; Alltech Associates, Inc., Deerfield, IL, USA). The separation of quercetin glucosides was performed based on a previous method, but with a more highly concentrated solvent (30\%) (19). The UV detection was monitored at a wavelength of $362 \mathrm{~nm}$. The system was set to maintain a 5 min post-run for re-equilibration between injections.

Standard preparation. 3,4'-Qdg, 4'-Qmg and 3-Qmg were dissolved in methanol $(1 \mathrm{mg} / \mathrm{ml})$ to prepare standard solutions, which were stored at $-20^{\circ} \mathrm{C}$ prior to use.

Sample preparation. Onions (Allium cepa L.), Welsh onions (Allium fistulosum L.), garlic (Allium sativum) and Chinese 


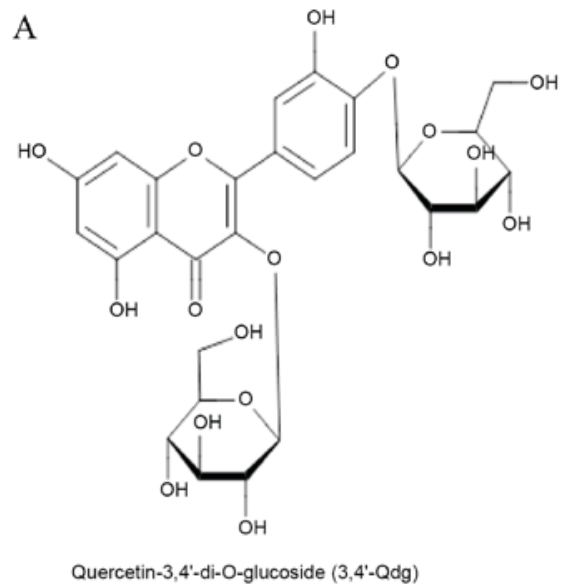

B

Quercetin-3,4'-di-O-glucoside (3,4'-Qdg)

$\mathrm{C}$

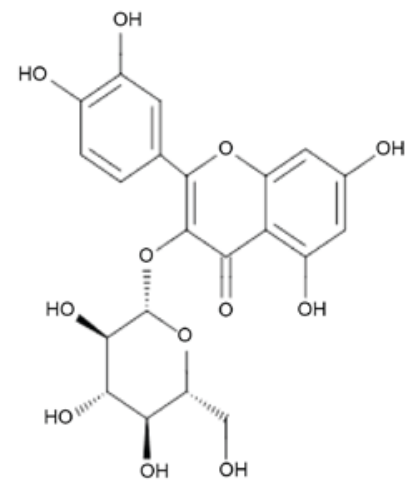

Quercetin-3-p-D-glucoside (3-Qmg)

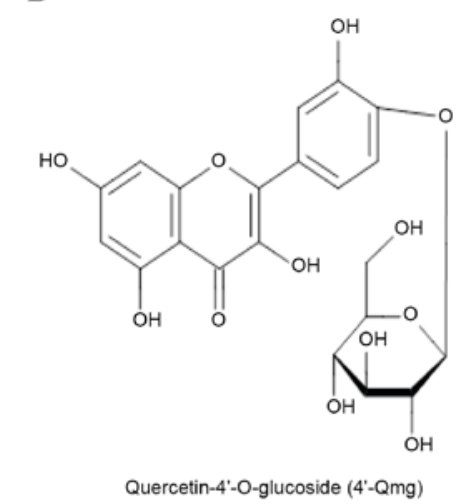

(n)

Figure 1. Chemical structures of (A) 3,4'-Qdg, (B) 4'-Qmg, (C) 3-Qmg and (D) rutin. 3,4'-Qdg, quercetin-3,4'-di-O-glucoside; 4'-Qmg, quercetin-4'-O-glucoside; 3-Qmg, quercetin 3- $\beta$-D-glucoside.

onions (Allium chinense) were purchased from a local market. The outer skins of the onions and garlic were removed and the bulbs $(10 \mathrm{~g})$ were sliced into small pieces and ground in an electric mixer. The bulb juices were frozen at $-80^{\circ} \mathrm{C}$ and lyophilized. Subsequently, the Chinese onions and welsh onions were divided into two parts, the stalk and the leaf. The Chinese onions and welsh onions were cleaned and cut into small pieces $(5 \mathrm{~g})$ and dried in a freeze drier, subsequent to being frozen at $-80^{\circ} \mathrm{C}$ overnight. In total, five fractions of dried samples were taken to be analyzed.

Sample extraction. The extraction of quercetin glucosides was performed according to a previous method (2). In total, $\sim 1 \mathrm{~g}$

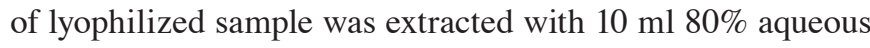
methanol and sonicated for $20 \mathrm{~min}$. The mixture was filtered through $0.45-\mu \mathrm{m}$ filter paper using a Büchner funnel under reduced pressure. Subsequently, $10 \mathrm{ml} 100 \%$ methanol was used to rinse the residue. The filtrates were pooled and mixed with $10 \mathrm{ml} 100 \%$ methanol. The mixture was concentrated using a rotary evaporator at $60^{\circ} \mathrm{C}$ for $30 \mathrm{~min}$. The remaining solvent $(3 \mathrm{ml})$ was mixed with $5 \mathrm{ml} \mathrm{100 \%}$ methanol to prepare a final volume of $10 \mathrm{ml}$ with Milli-Q purified water (Merck Millipore, Darmstadt, Germany). The solution was filtered through a $0.45 \mu \mathrm{m}$ polytetrafluoroethylene membrane filter and stored at $-20^{\circ} \mathrm{C}$ prior to analysis.
Cancer cell lines. The HepG2 cells (cat. no. HB-8065 ${ }^{\mathrm{TM}}$ ) were purchased from the American Type Culture Collection (ATCC; Rockville, MD, USA). The HepG2 cell line was sub-cultured and routinely grown with Dulbecco's modified Eagle's medium supplemented with 10\% FBS and 1\% streptomycin (Invitrogen; Thermo Fisher Scientific, Inc.) and incubated at $37^{\circ} \mathrm{C}\left(5 \% \mathrm{CO}_{2}\right.$ atmosphere). The complete growth medium was stored at $-4^{\circ} \mathrm{C}$ prior to analysis.

The PC-3 cells (cat. no. CRL-1435 ${ }^{\mathrm{TM}}$; ATCC ${ }^{\circledR}$ ) were purchased from ATCC (USA) and cultured in Ham's F-12K medium (Invitrogen; Thermo Fisher Scientific, Inc., Waltham, MA, USA) at $37^{\circ} \mathrm{C}$ in an atmosphere containing $5 \% \mathrm{CO}_{2}$. The HT-29 cells (cat. no., HTB-38 ${ }^{\mathrm{TM}}$ ) were purchased from the ATCC and cultured in Ham's F-12K medium at $37^{\circ} \mathrm{C}$ in an atmosphere containing $5 \% \mathrm{CO}_{2}$.

Cell viability assay. The effects of Allium cepa $L$. extracts and 3,4'-Qdg, 3-Qmg and 4'-Qmg on HepG2, PC-3 and HT-29 cell lines were determined in separate experiments. Cells were seeded at a density of 4,000 cells/well with complete growth medium in 96 -well plates and allowed to incubate at $37^{\circ} \mathrm{C}$ for $24 \mathrm{~h}$. Various concentrations of 3,4'-Qdg, 3-Qmg or 4'-Qmg in the growth medium were added into the wells, followed by $72 \mathrm{~h}$ incubation at $37^{\circ} \mathrm{C}$. An MTT assay (Thermo Fisher Scientific, Inc.) was conducted according to the manufacturer's 
Table I. Quercetin glucoside content of 6 vegetables of the genus Allium, as detected by high performance liquid chromatography.

\begin{tabular}{lccc}
\hline & \multicolumn{2}{c}{$\begin{array}{r}\text { Quercetin glucoside content, } \\
\mathrm{mg} / 100\end{array}$} \\
\cline { 2 - 4 } Allium species & $3,4^{\prime}-\mathrm{Qdg}$ weight & 3 -Qmg & 4'-Qmg \\
\hline Allium cepa L. & $714 \pm 47$ & $37 \pm 2.8$ & $619 \pm 19$ \\
Allium sativum & $\mathrm{ND}$ & $\mathrm{ND}$ & $\mathrm{ND}$ \\
Allium fistulosum L. leaf & $202 \pm 20$ & $\mathrm{ND}$ & $\mathrm{ND}$ \\
Allium fistulosum L. stalk & $472 \pm 3.6$ & $\mathrm{ND}$ & 42 \\
Allium chinense leaf & $77 \pm 2.2$ & $\mathrm{ND}$ & $\mathrm{ND}$ \\
Allium chinense stalk & $\mathrm{ND}$ & $\mathrm{ND}$ & $\mathrm{ND}$ \\
\hline
\end{tabular}

Data are expressed as the mean \pm standard deviation $(n=2) .3,4^{\prime}-\mathrm{Qdg}$, quercetin-3,4'-di-O-glucoside; 3-Qmg, quercetin 3- $\beta$-D-glucoside; 4'-Qmg, quercetin-4'-O-glucoside; ND, not detected.

Table II. Amounts of quercetin glucosides in the Allium genus, as detected by high performance liquid chromatography.

\begin{tabular}{lccc}
\hline & \multicolumn{2}{c}{$\begin{array}{c}\text { Quercetin glucoside content, } \\
\text { \% in Allium species }\end{array}$} \\
\cline { 2 - 4 } Allium species & $3,4^{\prime}$-Qdg & 3-Qmg & 4'-Qmg \\
\hline Allium cepa L. & 0.071 & 0.0037 & 0.062 \\
Allium sativum & $\mathrm{ND}$ & $\mathrm{ND}$ & $\mathrm{ND}$ \\
Allium fistulosum L. leaf & 0.020 & $\mathrm{ND}$ & $\mathrm{ND}$ \\
Allium fistulosum L. stalk & 0.047 & $\mathrm{ND}$ & 0.0042 \\
Allium chinense leaf & 0.0077 & $\mathrm{ND}$ & $\mathrm{ND}$ \\
Allium chinense stalk & $\mathrm{ND}$ & $\mathrm{ND}$ & $\mathrm{ND}$ \\
\hline
\end{tabular}

3,4'-Qdg, quercetin-3,4'-di-O-glucoside; 3-Qmg, quercetin 3- $\beta$-Dglucoside; 4'-Qmg, quercetin-4'-O-glucoside; ND, not detected.

protocol, in order to determine cell viability. Subsequent to the incubation, $20 \%(\mathrm{v} / \mathrm{v})$ MTT stock solution $(5 \mathrm{mg} / \mathrm{ml})$ was added to each well, followed by an additional $2-\mathrm{h}$ incubation at $37^{\circ} \mathrm{C}$. Subsequent to the removing and discarding of the supernatant, dimethyl sulfoxide was used to dissolve the converted formazan product with gentle agitation. Finally, a SpectraMax Plus 384 microplate reader (Molecular Devices, LLC, Sunnyvale, CA, USA) was applied to determine the absorbance at $540 \mathrm{~nm}$. The absorbance of the cells treated with quercetin glucosides was compared with that of the control cells to determine the relative cell viability.

\section{Results and Discussion}

Composition of quercetin glucosides content in vegetables. The composition of quercetin glucosides in the genus Allium was analyzed by HPLC and identified by mass spectrometry. Quercetin glucoside compounds were well separated by HPLC. The respective content of quercetin glucosides obtained from

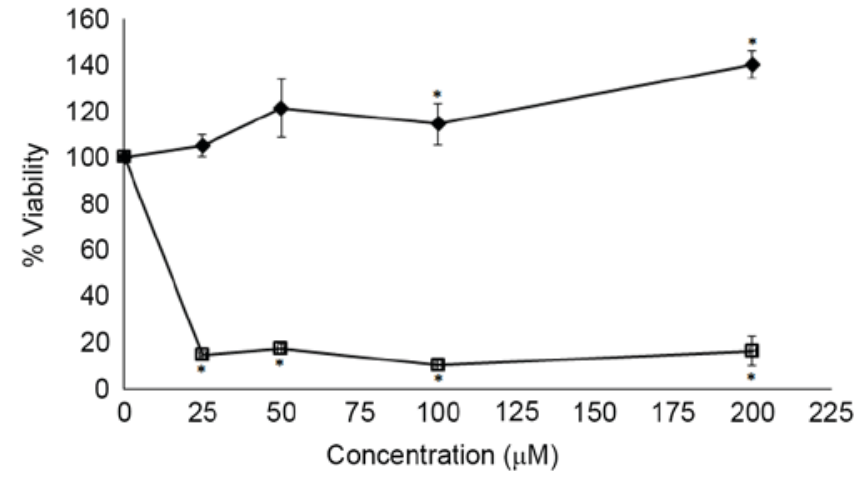

Figure 2. Effects of 3-Qmg and 4'-Qmg on cell viability of the HepG2 cancer cell line subsequent to a 72-h incubation. Data represent the mean \pm standard error of 3 experiments. ${ }^{*} \mathrm{P}<0.05$, compared with control. $\downarrow$, 3-Qmg; $\square, 4$ '-Qmg. 4'-Qmg, quercetin-4'-O-glucoside; 3-Qmg, quercetin 3- $\beta$-D-glucoside.

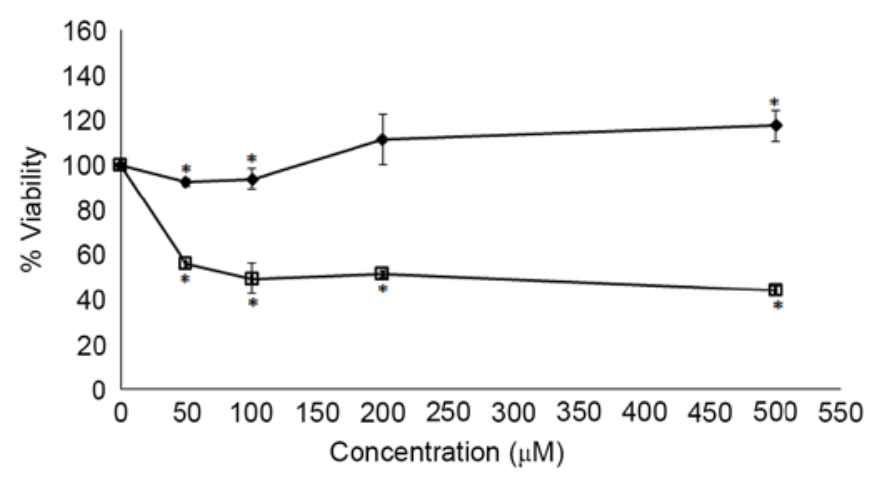

Figure 3. Effects of 3-Qmg and 4'-Qmg on the cell viability of the PC-3 cancer cell line subsequent to a 72-h incubation. Data represent the mean \pm standard error of 3 experiments. "P<0.05, compared with control. 3-Qmg; $\square, 4$ '-Qmg. 4'-Qmg, quercetin-4'-O-glucoside; 3-Qmg, quercetin 3- $\beta$-D-glucoside.

the methanolic extraction of Allium cepa $L$. was obtained by the HPLC analysis. The results reveal that 3-Qmg, 4'-Qmg and 3,4'-Qdg are the major quercetin glucosides present in Allium cepa L., which is consistent with previous studies (1-3). Other Allium varieties contained various amounts of quercetin glucosides (Table I). The composition of quercetin glucosides in genus Allium is presented in Table II.

Cytotoxic activity of quercetin glucosides. The effects of quercetin glucosides on cancer cell lines varied depending on the composition of quercetin glucosides in the vegetable extracts. The effects of Allium cepa L. extract on HepG2, HT-29 and PC-3 cancer cell lines were studied. The results revealed that the Allium cepa $L$. extract did not exhibit marked effects on cancer cell lines. However, individual quercetin glucosides exhibited significant effects $(\mathrm{P}<0.05)$ on HepG2 (Fig. 2), PC-3 (Fig. 3) and HT-29 cancer cell lines (Fig. 4) subsequent to $72 \mathrm{~h}$ of incubation. The present findings suggest that 4'-Qmg exhibited higher cytotoxic effects, compared with the other quercetins tested in the present study.

Activities of individual quercetin glucosides. Allium cepa $L$. extract contained a higher level of quercetin glucosides, as quantified by HPLC analysis (Table II). Varied compositions of quercetin glucosides among the Allium genus may 


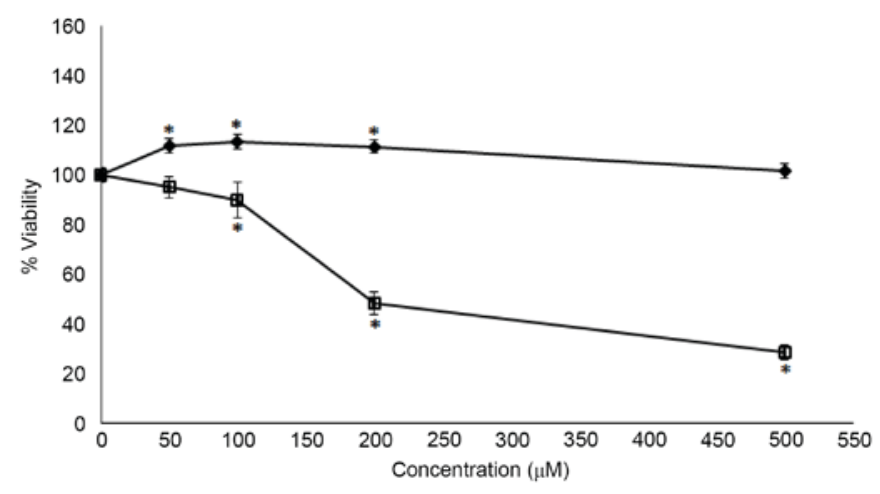

Figure 4. Effects of 3-Qmg and 4'-Qmg on cell viability of the HT-29 cancer cell line subsequent to a 72-h incubation. Data represent the mean \pm standard error of 3 experiments. ${ }^{*} \mathrm{P}<0.05$, compared with control. $\star$, 3-Qmg; $\square$, 4'-Qmg. 4'-Qmg, quercetin-4'-O-glucoside; 3-Qmg, quercetin 3- $\beta$-D-glucoside.

contribute to the variations in the anticancer properties of the extracts. Previous studies revealed the anticancer effects of quercetin glucosides in in vitro studies $(15,20,21)$. The present results indicate that quercetin glucosides are potent in HepG2, HT-29 and PC-3 cancer cell lines. The results are concordant with previous studies, which demonstrated that the Allium genus contains quercetins that exhibit health benefits in humans $(8-9,11)$. The major extractable quercetin glucosides in the Allium cepa L. were 3,4'-Qdg and 4'-Qmg, and 3'-Qmg was also detected in this species. The composition analysis of Allium cepa $L$ is presented in Table I, which indicates that 3,4'-Qdg is the major phenolic compound present in onions $(20,21)$. Certain quercetin glucosides may be cross-linked to cell-wall polysaccharides in the form of dimers that may be attributed to the quercetin glucosides level in the fraction obtained from different genus Allium (20,21).

Anti-proliferation of cancer cells. Treatment of various cancer cell lines with Allium extracts was performed. The content of quercetin glucosides extracted from Allium samples differed. The anticancer effect of quercetin glucosides was observed in different cancer cell lines with varied effects. The anti-proliferative effects of quercetin glucosides, obtained from onion extracts, on HepG2 subsequent to 72 -h incubation was studied (Figs. 2-4). The results are concordant with previous studies $(22,23)$, which suggested that Quercetin glucosides in food products have been reported to exhibit chemopreventive activities. The lowest $\mathrm{IC}_{50}$ value for onion extracts in cancer cell lines has been observed for those extracts with the highest presence of quercetin glucosides $(22,23)$. This indicates that quercetin glucosides may be the most potent bioactive compound in onion extracts. The findings of the present study also suggested that 4'-Qmg exhibits higher anti-proliferative activities, compared with other compounds in onion extracts, and only bioactive quercetin glucosides may exhibit inhibitory effects on cancer cells. The primary phenolic compounds in the methanolic extract were quercetin glucosides, including 3,4'-Qdg and 4'-Qmg (Table II). The results suggest that 3,4'-Qdg was an effective inhibitor on cell growth of HepG2, PC-3 and HT-29 cells. Quercetins have been reported to exhibit anti-proliferative activity towards cancer cells in vitro and in vivo $(15,20,21,23)$. Quercetin glucosides are considered to act as antioxidants in the genus Allium $(22,23)$. The combination of quercetin glucosides may contribute to the anti-proliferative activity of onion extracts on cancer cells (20-23). Among the quercetin glucosides present in onions, 4'-Qmg has been demonstrated to exhibit the most antioxidant activity in cancer cell lines (23). In addition, quercetin glucosides may function as apoptosis inducers in human cancer cells with inhibitory effects on proliferation (23).

\section{Conclusion}

In conclusion, methanol effectively isolated quercetin glucosides from the genus Allium, and the glucoside content varies with the species of Allium. The primary bioactive compound in the methanolic extract was 3,4'-Qdg, which is likely to be an important contributor to the anti-proliferative activity of the onions.

\section{Acknowledgements}

This study was supported by Keenway Industries Ltd. (grant no. 6903088).

\section{References}

1. Slimestad R, Fossen T and Vågen IM: Onions: A source of unique dietary flavonoids. J Agric Food Chem 55: 10067-10080, 2007.

2. Lee J and Mitchell AE: Pharmacokinetics of quercetin absorption from apples and onions in healthy humans. J Agric Food Chem 60: 3874-3881, 2012.

3. Bae CR, Park YK and Cha YS: Quercetin-rich onion peel extract suppresses adipogenesis by down-regulating adipogenic transcription factors and gene expression in 3T3-L1 adipocytes. J Sci Food Agric 94: 2655-2660, 2014.

4. Kiokias S and Varzakas T: Activity of flavonoids and $\beta$-carotene during the auto-oxidative deterioration of model food oil-in water emulsions. Food Chem 150: 280-286, 2014.

5. Williamson G, Plumb GW, Uda Y, Price KR and Rhodes MJ: Dietary quercetin glycosides: Antioxidant activity and induction of the anticarcinogenic phase II marker enzyme quinone reductase in Hepalclc7 cells. Carcinogenesis 17: 2385-2387, 1996.

6. Yang J, Meyers KJ, van der Heide J and Liu RH: Varietal differences in phenolic content and antioxidant and antiproliferative activities of onions. J Agric Food Chem 52: 6787-6793, 2004.

7. Lin S, Zhu Q, Wen L, Yang B, Jiang G, Gao H, Chen F and Jiang Y: Production of quercetin, kaempferol and their glycosidic derivatives from the aqueous-organic extracted residue of litchi pericarp with Aspergillus awamori. Food Chem 145: 220-227, 2014.

8. Zielińska M, Gülden M and Seibert H: Effects of quercetin and quercetin-3-O-glycosides on oxidative damage in rat C6 glioma cells. Environ Toxicol Pharmacol 13: 47-53, 2003.

9. Rohn S, Buchner N, Driemel G, Rauser M and Kroh LW: Thermal degradation of onion quercetin glucosides under roasting conditions. J Agric Food Chem 55: 1568-1573, 2007.

10. Caridi D, Trenerry VC, Rochfort S, Duong S, Laugher D and Jones R: Profiling and quantifying quercetin glucosides in onion (Allium cepa L.) varieties using capillary zone electrophoresis and high performance liquid chromatography. Food Chem 105: 691-699, 2007.

11. Wagner C, Fachinetto R, Dalla Corte CL, Brito VB, Severo D, de Oliveira Costa Dias G, Morel AF, Nogueira CW and Rocha JB: Quercitrin, a glycoside form of quercetin, prevents lipid peroxidation in vitro. Brain Res 1107: 192-198, 2006.

12. Yang WS, Jeong D, Yi YS, Lee BH, Kim TW, Htwe KM, Kim YD, Yoon KD, Hong S, Lee WS and Cho JY: Myrsine seguinii ethanolic extract and its active component quercetin inhibit macrophage activation and peritonitis induced by LPS by targeting to Syk/Src/IRAK-1. J Ethnopharmacol 151: 1165-1174, 2014. 
13. Sugantha Priya E, Selvakumar K, Bavithra S, Elumalai P, Arunkumar R, Raja Singh P, Brindha Mercy A and Arunakaran J: Anti-cancer activity of quercetin in neuroblastoma: An in vitro approach. Neurol Sci 35: 163-170, 2014.

14. Cao HH, Tse AK, Kwan HY, Yu H, Cheng CY, Su T, Fong WF and Yu ZL: Quercetin exerts anti-melanoma activities and inhibits STAT3 signaling. Biochem Pharmacol 87: 424-434, 2014.

15. Žižková P, Blaškovič D, Májeková M, Svorc L, Račková L, Ratkovská L, Veverka M and Horáková L: Novel quercetin derivatives in treatment of peroxynitrite-oxidized SERCA1. Mol Cell Biochem 386: 1-14, 2014.

16. Hollman PC, de Vries JH, Van Leeuwen SD, Mengelers MJ and Katan MB: Absorption of dietary quercetin glycosides and quercetin in healthy ileostomy volunteers. Am J Clin. Nutr 62: 1276-1282, 1995.

17. Hollman PC, van Trijp JM, Mengelers MJ, de Vries JH and Katan MB: Bioavailability of the dietary antioxidant flavono quercetin in man. Cancer Lett 114: 139-140, 1997.

18. Marotti M and Piccaglia R: Characterization of flavonoids in different cultivars of onion (Allium cepa L.). J Food Sci 67 1229-1232, 2002.
19. Lu X, Ross CF, Powers JR and Rasco BA: Determination of quercetins in onion (Allium cepa) using infrared spectroscopy. J Agric Food Chem 59: 6376-6382, 2011.

20. Yang HH, Hwangbo K, Zheng MS, Cho JH, Son JK, Kim HY, Baek SH, Choi HC, Park SY and Kim JR: Quercetin-3-O- $\beta$-D-glucuronide isolated from Polygonum aviculare inhibits cellular senescence in human primary cells. Arch Pharm Res 37: 1219-1233, 2014.

21. Li W, Liu M, Xu YF, Feng Y, Che JP, Wang GC and Zheng JH: Combination of quercetin and hyperoside has anticancer effects on renal cancer cells through inhibition of oncogenic microRNA-27a. Oncol Rep 31: 117-124, 2014.

22. Chen JY, Hu RY and Chou HC: Quercetin-induced cardioprotection against doxorubicin cytotoxicity. J Biomed Sci 20: 95, 2013.

23. Chang YC, Tsai MH, Sheu WH, Hsieh SC and Chiang AN: The therapeutic potential and mechanisms of action of quercetin in relation to lipopolysaccharide-induced sepsis in vitro and in vivo. PLoS One 8: e80744, 2013. 